\title{
Superinsulator Phase of two-dimensional Superconductors
}

\author{
A. Krämer and S. Doniach \\ Dept. of Applied Physics, Stanford University, Stanford CA 94305, U.S.A.
}

(July 22, 1998)

\begin{abstract}
Using path-integral Quantum Monte Carlo we study the low-temperature phase diagram of a two-dimensional superconductor within a phenomenological model, where vortices have a finite mass and move in a dissipative environment modeled by a Caldeira-Leggett term. The quantum vortex liquid at high magnetic fields exhibits superfluidity and thus corresponds to a superinsulating phase which is characterized by a nonlinear voltage-current law for an infinite system in the absence of pinning. This superinsulating phase is shifted to higher magnetic fields in the presence of dissipation.
\end{abstract}

74.60.Ge, 74.76.-w

Typeset using REVTEX 
Quantum fluctuations of vortices play an important role for the low-temperature physics of two-dimensional (2D) superconductors. It has been established that dissipative [1], inertial [2]:3], or Hall-type [4] zero point motion is able to melt the vortex lattice at sufficiently high magnetic fields and give way to a quantum vortex liquid phase in principle at $T=0$. In the presence of disorder this melting transition is a continuous one and corresponds to the localization or crystallization of Cooper pairs in the theory of the superconductor-insulator transition [5.6]. However, for a system without disorder, the melting of the vortex lattice is discontinuous in $(2+1)$ dimensions [3].

In this letter, using path-integral Quantum Monte Carlo (QMC), we will study a phenomenological model where the vortices have a finite mass and move in a dissipative environment, which is modeled by a Caldeira-Leggett term. Both, vortex mass and dissipation are assumed to originate from electronic contributions while the vortices themselves are considered as bosons. For the simulations we focus on the situation where the Magnus force is zero which is appropriate for the case of granular systems or Josephson Junction arrays (JJA) where experiments suggest that the transverse force is very small [7]. The effect of a weak Magnus force will then be discussed afterwards.

In a thin film superconductor at high magnetic fields vortices effectively interact logarithmically. So the question arises whether the vortices can form a superfluid or not. We will show using QMC that a 2D system of logarithmically interacting particles indeed exhibits superfluidity at low temperatures and high densities even in the presence of dissipation. 8] This means that the quantum vortex liquid actually corresponds to a superinsulating phase in which an infinitesimal current will cause an infinite voltage in the absence of pinning effects in analogy with sliding charge density waves. This superinsulating phase exhibits a nonlinear voltage-current law with non-universal exponent and is different from the insulating Cooper pair glass phase in Fisher's [5] treatment of the field-tuned superconductor-insulator transition in the presence of strong disorder. We will show numerically that at finite temperature the superinsulator gives way to a classical vortex liquid via a Kosterlitz-Thouless (KT-) transition. In the presence of dissipation this phase transition is shifted to higher magnetic fields possibly giving rise to an intermediate non-superfluid vortex liquid phase at $T=0$.

In the non-dissipative case the vortex system is described by the Euclidean Lagrangian

$$
\mathcal{L}=\frac{m}{2} \sum_{k}\left(\frac{d \mathbf{r}_{k}}{d \tau}\right)^{2}-T_{0} \sum_{k>l} \ln \left(\left|\mathbf{r}_{k}-\mathbf{r}_{l}\right|\right),
$$

where $\mathbf{r}_{k}(\tau)$ are the vortex positions, $m$ is the vortex mass, and $T_{0}$ controls the strength of the logarithmic interactions. We assume the system to be embedded in a neutralizing background which corresponds to the presence of a uniform external magnetic field $B=\Phi_{0} \varrho$ so that the long-range interaction in Eq. (1) is well-defined even for periodic boundary conditions. Here, $\varrho$ is the vortex density, and $\Phi_{0}$ is the flux quantum. $\mathbf{r}_{k}(\tau)$ obeys periodic boundary conditions in imaginary time $\tau$, that include bosonic exchange of the particles.

Finite-temperature QMC simulations have been performed for systems with up to 128 time slices and $\mathrm{N}=16,28$ and 36 particles on a grid of $256 \times 222$ sites in a rectangular periodic box which was commensurable with a triangular vortex lattice. A bisection algorithm was used where several particles are updated in several imaginary time slices simultaneously in order to allow for cutting and reconnection of the paths [14]. The superfluid density $\varrho_{s}$ was 
then obtained from the distribution of winding numbers $W_{i},(i=x, y)$ around the periodic cell by 15

$$
\frac{\varrho_{s}}{\varrho}=\frac{m L_{i}^{2}}{\beta \hbar^{2} N}\left\langle W_{i}^{2}\right\rangle
$$

where $L_{i}$ is the periodic box length in $i$-direction, and $\beta$ is the inverse temperature. For the lowest-temperature data reported in this work typically about $10^{5}$ sweeps were needed to equilibrate the winding number moves. Equilibration was checked by carefully monitoring the distribution of $W_{i}$ which can be fitted by a Gaussian at low $T$. In the following we use dimensionless variables, where the temperature $T$ is given in units of $T_{0}$, and the magnetic field $B$ is given in units of $B_{0}=\left(m T_{0} \Phi_{0}\right) /\left(\sqrt{3} \hbar^{2}\right)$.

The phase diagram of the model (1) is shown in Fig. 1a. It consists of three phases: a superconducting vortex solid phase (VS) at low densities and temperatures, a classical vortex liquid (VL) at high temperatures, and a quantum vortex liquid (QVL) at high densities and low temperatures. The vortex solid thermally melts via a KT-transition mediated by the unbinding of dislocation pairs which takes place at $T_{K T} \approx 0.0071$ [16] and is approximately independent of the magnetic field $B$. At low temperatures quantum fluctuations melt the vortex solid via a discontinuous transition at the melting field $B_{M} \approx 0.0077$ [2] which is approximately independent of the temperature [3]. In order to check our numerical algorithm we measured the internal energy at various fields and low temperature, and also estimated $B_{M}$ by calculating the Bragg-peak intensity. The results where found to be in good quantitative agreement with the zero temperature results reported in Ref. [2].

The inset in Fig. 1a shows the superfluid fraction $\varrho_{s} / \varrho$ as function of the magnetic field $B$ at $T=0.005$ as obtained in our simulations. At the quantum melting transition $\varrho_{s} / \varrho$ jumps from zero to one which quantitatively reproduces the result of Nordborg and Blatter [9], and shows that the QVL actually is a vortex superfluid with $\varrho_{s}=\varrho$. In the following we will focus on the transition from the QVL phase to the VL phase. The behavior of the superfluid fraction is shown in Fig. 2a where $\varrho_{s} / \varrho$ is plotted as function of the temperature $T$ for different magnetic fields $B$. It is seen that all data collapse onto one single curve when plotted against the scaling variable $T / B$. This scaling behavior can be understood with a KT-type unbinding of topological (vortex-antivortex) excitations (which we will call dual vortices) in the vortex-superfluid. Since the energy scale of the logarithmic interaction of dual vortices is given by $T_{0}^{\prime}=2 \pi \hbar^{2} \varrho / m$, the transition temperature thus scales with $B$. Because of the fundamental duality between vortices and charges [10] the dual vortices are actually related to the Cooper pair degrees of freedom in the system as will be discussed below.

Following Ceperley and Pollock [17] further numerical evidence for the existence of a KT-transition can be obtained by performing a finite-size analysis that explicitly invokes the KT-recursion relations [18] which are integrated up to the system radius $L / 2$. There are only two independent fit parameters for all numerical data involved in this procedure: the dual vortex core energy $E_{0}$ and the dual vortex radius $d$. The resulting fits are the solid curves in Fig.s 2a and 2b. Fig. 2b shows data for $N=16,28,36$, and also extrapolated to $N=\infty$. From this the KT-transition temperature is obtained as $T_{K T}^{\prime}=1.451 \hbar^{2} \varrho / \mathrm{m}$, where $E_{0}=6.49 \hbar^{2} \varrho / m$, and $d=0.634 \varrho^{-1 / 2}$. Interestingly it is observed that the core size $d$ scales with the vortex spacing $\varrho^{-1 / 2}$ which is a consequence of the logarithmic interactions which provide no additional length scale. 
In order to interpret these numerical results we note that the vortex superfluid actually should be considered as a charged superfluid where the flux quantum $\Phi_{0}$ plays the role of a "charge" and the "flux quantum" of the dual vortices is given by $2 e$. In a homogeneous superconductor the corresponding gauge field $\mathbf{a}$ is related to the 2D-superfluid density of Cooper pairs $n_{s}, \nabla \times \mathbf{a}=2 e n_{s}$, which gives rise to the Magnus force acting on the vortices [11]. To be consistent with the assumption of of a zero transverse force in a granular system we therefore suppose that $n_{s}$ is replaced by the density of fluctuating charges $\delta n_{s}$ on the superconducting islands, $\nabla \times \mathbf{a}=2 e \delta n_{s}$, which is a usual assumption in the context of granular superconductors or JJA's [12]. Static fluctuations of the gauge field a can approximately be included by a contribution $\left(\Lambda / 4 e^{2}\right)(\nabla \times \mathbf{a})^{2}$ to the free energy of the vortex superfluid, where $\Lambda$ is the strength of the $(\delta-)$ interaction between the Cooper pairs [22]. The numerical simulations then correspond to the case of strong repulsion, $\Lambda=\infty$, where $\delta n_{s}$ decays logarithmically around a dual vortex. For finite $\Lambda$ the vortex-superfluid current flowing around the dual vortices is screened on the length $\lambda=\left(2 \Lambda m / \hbar^{2} \varrho\right)^{1 / 2}$. In the following we assume that this screening length is much larger than the dual vortex core size $d$.

Let us discuss the dynamics of the vortex superfluid in the case $\Lambda=\infty$. Since dual vortex excitations carry a charge $\pm 2 e$ the separation of dual vortex-antivortex pairs leads to an electric current $J$. However, in an infinite system this process requires an infinite amount of energy when the electric field $E$ is zero, while for a non-zero electric field the energy barrier is finite. It is well known [19] that for $T \ll T_{K T}^{\prime}$ this type of dynamics leads to a non-linear voltage-current law with nonuniversal exponent,

$$
E \sim J^{T / T_{0}^{\prime}} .
$$

Eq. (3) explicitly shows that the vortex superfluid phase actually is a superinsulating phase with infinite resistivity even at non-zero temperature. In a realistic system finite-size effects as well as a finite screening length $\lambda$ will restore a finite resistivity $\rho \sim e^{E_{0} / T}$ for small currents where the Arrhenius factor $e^{-E_{0} / T}$ controls the number of dual vortex-antivortex pairs in the system, and the KT-transition will be broadened.

Let us now briefly consider the case where a weak Magnus force is present. This means, that the vortices on average pick up a phase $\phi=2 \pi n_{s}^{0} \xi_{0}^{2} \neq 0$ when encircling a superconducting grain of area $\xi_{0}^{2}$ [13]. Here, $2 e n_{s}^{0}$ is the charge density of Cooper pairs contributing to the transverse force which plays the role of a fictitious external magnetic field. By analogy with an ordinary superconductor we therefore expect that dual vortices will form an Abrikosov lattice. Thermal excitations and dynamics of this dual vortex lattice will then be governed by the unbinding of dislocation pairs and the KT-melting temperature is given by $T_{K T}^{\prime \prime} \approx 0.06 \hbar^{2} \varrho / m$. This again is a superinsulating phase described by the non-linear voltage-current law (3), where $T_{0}^{\prime}$ is replaced by the interaction energy of dislocations. The corresponding phase diagram is schematically shown in Fig. 1 b.

In what follows we will discuss the influence of dissipation, which is treated approximately by adding a Caldeira-Leggett term (time-delay) [20]

$$
\mathcal{L}_{C L}=-\frac{\eta}{2 \pi} \sum_{k} \int_{0}^{\hbar \beta} d \tau^{\prime} \frac{d \mathbf{r}_{k}}{d \tau} \ln \left|\sin \frac{\pi}{\hbar \beta}\left(\tau-\tau^{\prime}\right)\right| \frac{d \mathbf{r}_{k}}{d \tau^{\prime}}
$$


to the Lagrangian (11). Eq. (4) phenomenologically describes the coupling of each single vortex to a separate harmonic oscillator heat bath which is characterized by a frictional constant $\eta$. This model is appropriate to describe dissipation originating from normal currents in the vortex cores [1] and has also been used by Wheatley [21] to study the statistics of holons coupled to the background of spinons in the context of high- $T_{c}$ superconductivity. With regard to superfluidity there is one essential assumption in using Eq. (国) to model a dissipative environment. The coupling to independent harmonic oscillator heat baths cannot exactly be valid for indistinguishable particles. Following [21] we therefore assume that dependencies between separate heat baths induced by bosonic exchange of the vortices can be neglected.

We again computed the superfluid fraction which is shown in Fig. 3 for $B=0.04,0.08$, and $\eta=0.01,0.02$, where $\eta$ is given in units $\eta_{0}=\pi m T_{0} / \hbar$. It is seen that dissipation leads to a shift of the KT-transition to lower temperatures and, more surprisingly, to the suppression of superfluidity at $T=0$. This reentrance behavior can be understood by the contribution of the Caldeira-Leggett term (14) to the action of vortices involved in a particle exchange, which can be described in terms of a temperature-dependent effective mass, $m^{*}=m+\beta \hbar \eta(\ln 2 / \pi)$. For the bare vortex superfluid density $\tilde{\varrho}_{s}$ (unrenormalized by dual vortex-antivortex pairs) we therefore make the ansatz $\tilde{\varrho}_{s}=\varrho\left(\mathrm{m} / \mathrm{m}^{*}\right)$, which is a linear function of $T$ at low temperatures. Since the interaction of dual vortices scales with $\tilde{\varrho}_{s}$, the KT-transition is then shifted to lower temperatures, $T_{K T}^{\prime, \eta}=T_{K T}^{\prime}-(\eta \hbar / m)(\ln 2 / \pi)$. Using this ansatz and taking the core-energy to be $E_{0}^{\eta}=E_{0} \tilde{\varrho}_{s} / \varrho$ we perform the same finite-size fit as above which yields the solid curves in Fig. 3 and shows a remarkable coincidence with the numerical data. Again the same values for $E_{0}$ and $d$ as before were used so that no new fit parameters appear here. The dashed curves in Fig. 3 show the superfluid fraction extrapolated for $N=\infty$. At low temperature, $\varrho_{s} / \varrho$ rises linearly with $T$ and reaches its maximum value $\approx 1-0.15 \eta /(\hbar \varrho)$ near $T_{K T}^{\prime, \eta}$.

The KT-transition line between the VL and the QVL (extrapolated to $T=0$ ) is shown in the $B$-T-diagram in the inset of Fig. 3 where the friction parameter $\eta$ was assumed to be independent of the temperature. The crossing point with the $B$-axis $\left(T_{K T}^{\prime, \eta}=0\right)$ is given by $B_{K T}^{0} \approx 0.15 \eta \Phi_{0} / \hbar$. If $B_{K T}^{0} \gtrsim B_{M}$ this suggests the existence of an intermediate field range in which the classical vortex liquid phase continues to $T=0$. [23] In this regime the superinsulating phase is unstable against the unbinding of dual vortex-antivortex pairs even at $T=0$ since the strength of the logarithmic dual vortex-vortex interaction is proportional to $T$. This also means that the exponent of the voltage-current law (3) will stay finite in the limit $T \rightarrow 0$.

We add a few comments about the experimental accessibility of the results presented in this paper. The notion of a quantum melting transition as it was treated here requires a system with weak pinning as well as the existence of a finite vortex mass which has to be small enough in order to push the melting field $B_{M}$ significantly below the upper critical field $B_{c 2}$. Indeed, a field-tuned superconductor-insulator transition which can be related to the melting of the vortex lattice has been observed in Josephson junction arrays [24]. According to our results for a finite system the conductivity in the superinsulating phase is expected to show an Arrhenius behavior $\sigma \sim e^{-E_{0} / T}$ where dissipation (as it was modeled here) leads to a linear $T$-dependence of $E_{0}$ at low temperatures so that the conductivity stays finite in the limit $T \rightarrow 0$. This is qualitatively consistent with the experimental results in Ref. [24]. 
In conclusion, using QMC simulations, we have studied superfluidity in a system of logarithmically interacting massive vortices moving in a dissipative environment. It was shown that the quantum vortex liquid at high magnetic fields corresponds to a superinsulating phase characterized by a non-linear voltage-current law with non-universal exponent. This superinsulating phase gives way to a classical vortex-liquid phase via a Kosterlitz-Thouless transition, which is shifted to higher magnetic fields in the presence of dissipation.

\section{ACKNOWLEDGMENTS}

We thank D. Das for useful discussions. We also gratefully acknowledge financial support by the Deutsche Forschungsgemeinschaft and the NSF under grant No. DMR 9627459. Numerical work has partly been done at the San Diego Supercomputer Center (SDSC). 


\section{REFERENCES}

[1] G. Blatter et al., Phys. Rev. B 50, 13013 (1994).

[2] W.R. Magro and D.M. Ceperley, Phys. Rev. B 48, 411 (1993).

[3] T. Onogi and S. Doniach, Sol. State Comm. 98, 1 (1996).

[4] A. Rozhkov and D. Stroud, Phys. Rev. B 54, R12697 (1996).

[5] M.P.A. Fisher, Phys. Rev. Lett. 65, 923 (1990).

[6] S. Doniach and M. Inui, Phys. Rev. B 41, 6668 (1990).

[7] S.G. Lachenmann et al., Phys. Rev. B 50, 3158 (1994); H.S.J. van der Zant al., Phys. Rev. B 50, 340 (1994); H.S.J. van der Zant al., Europhys. Lett. 18,343 (1992).

[8] Recently Nordborg and Blatter [9] have done a similar calculation for studying the entanglement of 3D classical vortices at high temperatures.

[9] H. Nordborg and G. Blatter, Phys. Rev. Lett. 79, 1925 (1997).

[10] D.H. Lee and M. Fisher, Phys. Rev. B 39, 2756 (1988); X.G. Wen and A. Zee, Phys. Rev. Lett. 62, 1937 (1989).

[11] P. Ao and D.J. Thouless, Phys. Rev. Lett. 70, 2158 (1993).

[12] Y.M. Blanter, R. Fazio, and G. Schön, Nuclear Physics B, Proc. Suppl. 58,79 (1997); R. Fazio et al., Helv. Phys. Acta 65, 228 (1992).

[13] X.-M. Zhu, Y. Tan, and P. Ao, Phys. Rev. Lett. 77, 562 (1996).

[14] K.E. Schmidt and D.M. Ceperley, in The Monte Carlo method in condensed matter physics edited by K. Binder, Springer, Berlin Heidelberg 1992.

[15] E.L. Pollock and D.M. Ceperley, Phys. Rev. B 36, 8343 (1987).

[16] B.A. Huberman and S. Doniach, Phys. Rev. Lett. 43, 950 (1979); D.S. Fisher, Phys. Rev. B 22, 1190 (1980).

[17] D.M. Ceperley and E.L. Pollock, Phys. Rev. B 39, 2084 (1989).

[18] J.M. Kosterlitz und D.J. Thouless, J. Phys. C 6, 1181 (1973).

[19] J.M. Kosterlitz and D.J. Thouless in Progress in low temperature physics, Vol. VIIb, edited by D.F. Brewer, Amsterdam 1978.

[20] A.O. Caldeira und A.J. Leggett, Ann. Phys. (N. Y.) 149, 374 (1983).

[21] J.M. Wheatley, Phys. Rev.B 41, 7301 (1990).

[22] In a JJA the parameter $\Lambda$ is related to the charging energy $E_{C}$.

[23] We note that a non-zero friction constant $\eta$ also shifts the melting field $B_{M}$ itself to higher values. However, our simulation results indicate that this shift is smaller than the shift of the superinsulating phase.

[24] H.S.J. van der Zant et al., Phys. Rev. Lett. 69, 2971 (1992). 


\section{FIGURES}

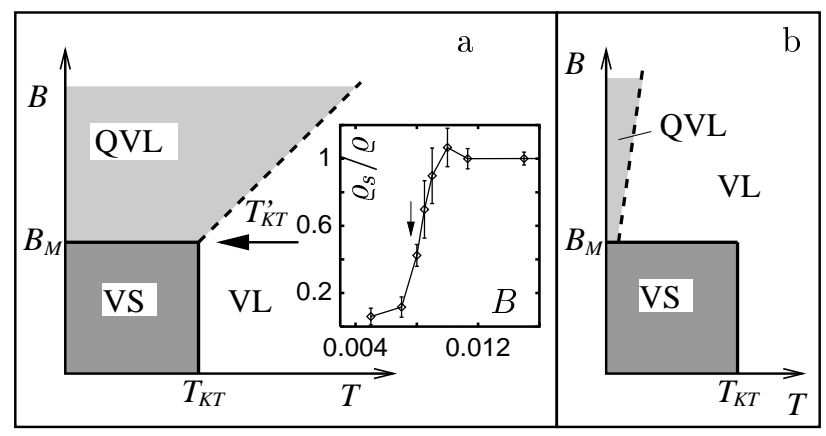

FIG. 1. ( $B-T)$-phase diagram of the vortex system in a $2 \mathrm{D}$ superconductor (below $\left.B_{c 2}\right)$. The inset shows the jump in the superfluid density at the quantum melting transition at $B_{M} \approx 0.0077$ (arrow). (a) zero Magnus force. (b) weak Magnus force.

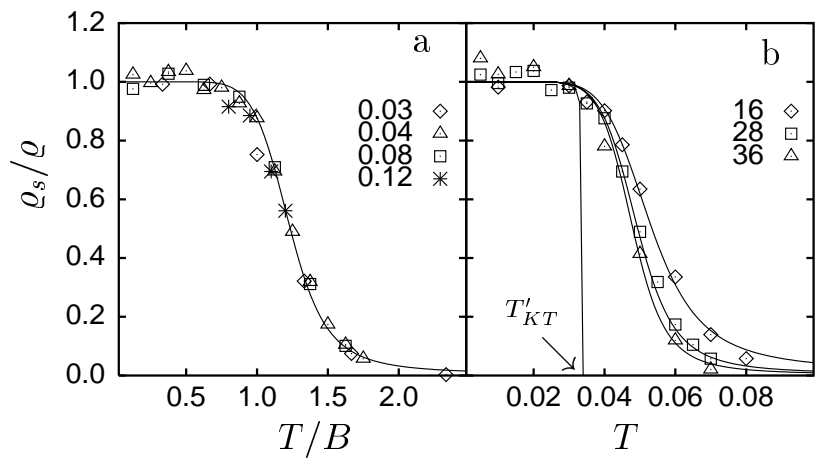

FIG. 2. Superfluid fraction $\varrho_{s} / \varrho$ : (a) as function of the scaling variable $T / B$ for the magnetic fields $B=0.03,0.04,0.08$, and 0.12 , and a 28-particle system. (b) at $B=0.04$ as function of $T$ for systems with 16, 28, and 36 particles and extrapolated for an infinite system.

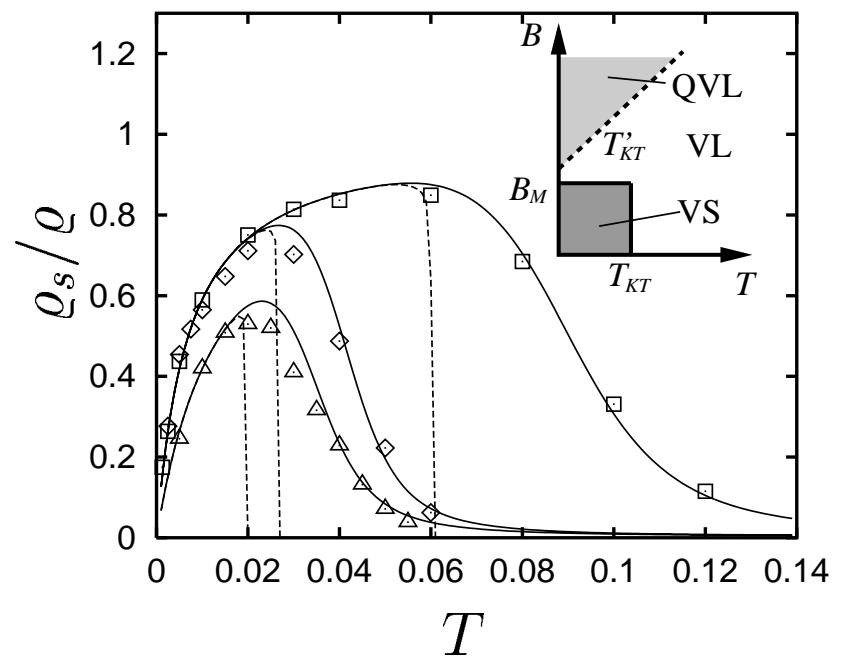

FIG. 3. Superfluid fraction $\varrho_{s} / \varrho$ as function of $T$ for non-zero dissipation $(N=28): B=0.04$, $\eta=0.01(\diamond), B=0.04, \eta=0.02(\triangle)$, and $B=0.08, \eta=0.01(\square)$. Dashed curves: Finite-size extrapolation to $N=\infty$. Inset: $(B-T)$-phase diagram (schematically). 